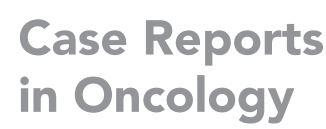

\title{
Immune Checkpoint Inhibitor-Induced Polymyositis and Myasthenia Gravis with Fatal Outcome
}

\author{
Daniel Giglio $^{a}$ Henrik Berntsson $^{b}$ Åsa Fred ${ }^{c}$ Lars $\mathrm{Ny}^{\mathrm{a}, \mathrm{d}}$ \\ aDepartment of Oncology, Institute of Clinical Sciences, Sahlgrenska Academy at the \\ University of Gothenburg, Gothenburg, Sweden; 'bepartment of Medicine, Halland \\ Hospital Varberg, Varberg, Sweden; 'Department of Pathology, Halland Hospital \\ Halmstad, Halmstad, Sweden; dDepartment of Oncology, Sahlgrenska University Hospital, \\ Gothenburg, Sweden
}

\author{
Keywords \\ Colorectal adenocarcinoma - Hepatocellular carcinoma - Microsatellite-instable tumor . \\ Pembrolizumab · Myositis · Myasthenia gravis
}

\begin{abstract}
We here report on a 74-year-old man diagnosed with a pT3CNO BRAF-mutated and mismatch repair-deficient adenocarcinoma in the colon ascendens and 3 liver metastases. After hemicolectomy, the patient received treatment with the PD-1 inhibitor pembrolizumab. Three weeks later (on day 22), laboratory tests showed leukocytosis and an increase in transaminases; immune checkpoint inhibitor $(\mathrm{ICl})$-induced hepatitis was suspected and prednisolone therapy was initiated. On day 29, the patient was acutely hospitalized due to dyspnea, somnolence and walking difficulties. Dysarthria, hoarseness, muscle pain and weakness had developed and the dose of prednisolone was increased. Serum levels of lactate dehydrogenase, creatine kinase and myoglobin were increased and $\mathrm{ICl}$-induced myositis was suspected. Antibodies against acetylcholine receptor and titin were present, indicating myasthenia gravis. Eventually, bulbar myopathy developed, including dysarthria and dysphagia, and the patient could no longer attain saturation without oxygen. The patient was transferred to the intensive care unit, intubated and given methylprednisolone, intravenous immunoglobulins and infliximab. The patient developed carbon dioxide retention and died on day 39. Microscopical examination of the intercostal musculature, diaphragm, cervical musculature and tongue showed inflammatory infiltration and fibrosis consistent with a pronounced myositis. In the liver, microscopical examination did not show metastases from colorectal cancer but instead a hepa-
\end{abstract}


tocellular cancer. The cause of death was determined as respiratory insufficiency due to polymyositis. In conclusion, ICls may induce myositis combined with neurological immune-related adverse events. In patients developing muscle weakness and pain under ICl therapy, myositis should be suspected.

(C) 2020 The Author(s).

Published by S. Karger AG, Basel

\section{Introduction}

The interaction between programmed cell death ligand 1 (PD-L1) on tumor cells and programmed cell death 1 (PD-1) expressed by cytotoxic T lymphocytes leads to T-cell exhaustion and tumor immune escape [1]. The use of immune checkpoint inhibitors (ICIs) targeting the interaction between PD-1 and PD-L1 has led to a paradigm shift in the prognosis of stage IV melanoma. The use of ICIs has been expanded to many other solid tumor forms including renal cancer, urinary bladder cancer, lung cancer and colorectal cancer. Pembrolizumab is a humanized antibody targeting PD-1 approved for the treatment of melanoma and many solid tumors including certain tumors of the gastrointestinal tract. PD-L1 is, however, not only expressed on tumor cells but also on cells in different tissues of the body. Patients treated with ICIs may therefore experience immune-related adverse events (irAEs) of variable severity. The most common immune-related side effects occur in the endocrine system, skin, and pulmonary and gastrointestinal tract. irAEs affecting the nervous system are rare and comprise conditions such as Guillain-Barré syndrome, myasthenia gravis (MG), aseptic meningitis, transverse myelitis and immune encephalitis [2]. Myositis may also occur and may be accompanied by MG [3].

Here, we report on a patient experiencing a combination of ICI-induced myositis and neurological irAEs, having subtle initial symptoms but a dramatic and fatal course.

\section{Case Report}

A 74-year-old man with hypertension and insulin-dependent diabetes mellitus type 2 including diabetic retinopathy was referred to the surgery department due to an 8-kg weight loss and iron deficiency anemia. Computed tomography and magnetic resonance imaging displayed a tumor in the colon ascendens and 3 liver metastases $(15,20$ and $65 \mathrm{~mm}$ in dimension). The patient underwent right hemicolectomy. The pathology report showed a $60 \times 70 \times 50 \mathrm{~mm}$ low-grade pT3cN0 adenocarcinoma, absence of metastases in 24 excised lymph nodes and presence of lymphovascular growth but no vascular or perineural growth. The tumor had an activated BRAF mutation in exon 15 (V600E) and loss of expression of MLH1 and PMS2 and was defined as a mismatch repair-deficient (MMR-D)/microsatelliteinstable (MSI) tumor. Since the patient had a nonfavorable prognostic profile for response to chemotherapy but fulfilled tumor criteria for a high chance of response to immunotherapy, the patient initiated therapy with the PD-1 inhibitor pembrolizumab (Keytruda ${ }^{\circledR} ; 200-\mathrm{mg}$ intravenous flat dose) in order to potentially be eligible for liver metastasectomy.

On day 7 after the first infusion of pembrolizumab, the patient reported having symptoms of a cold but was otherwise asymptomatic. A laboratory test showed leukocytosis (white blood cell count $13.49 \times 10^{9} / \mathrm{L}$ ) and a slight increase in C-reactive protein (24 mg/L; Fig. 1a). The cold was still present on day 22 , when he was planned for the second cycle of pembrolizumab. The patient now reported dry coughing, had no fever and was otherwise asymptomatic. A laboratory test showed an increase in AST and ALT ( $>3 \times$ ULN; Fig. 1b) and was judged to have ICI-induced hepatitis grade 2 , and the patient therefore initiated prednisolone

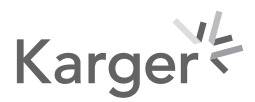




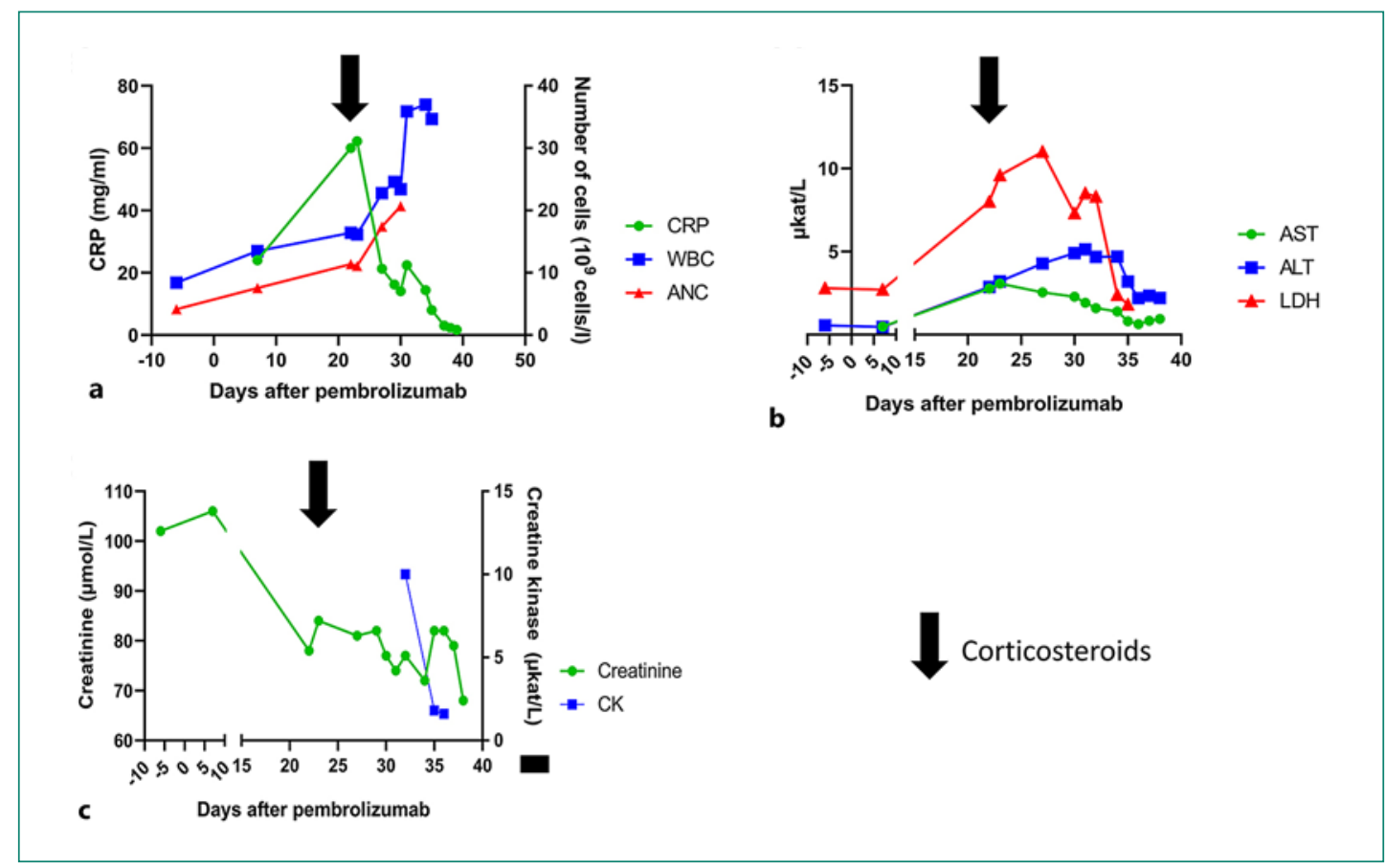

Fig. 1. Changes in plasma levels of C-reactive protein (CRP), white blood cell count (WBC) and absolute neutrophil count (ANC) (a), of aspartate aminotransferase (AST), alanine aminotransferase (ALT) and lactate dehydrogenase (LDH) (b), and of creatinine and creatine kinase (CK) (c) during the clinical course.

therapy (50 mg once daily), which resulted in a decrease in C-reactive protein and AST, but white blood cells and neutrophils were increased (Fig. 1b). The second dose of pembrolizumab (on day 22) was not given. On day 29 , the patient was acutely hospitalized due to dyspnea. Initially, myocardial infarction was suspected due to an elevation of troponin T (482 $\mathrm{ng} / \mathrm{L}$ ); echocardiography showed septal hypokinesia, but troponin $\mathrm{T}$ did not show any dynamic change over time. The patient developed somnolence and had difficulty walking. On day 30, a clinical examination revealed that the patient had developed dysarthria and hoarseness. The patient complained about pain in his neck and right leg and had difficulty raising his right leg. The dose of prednisolone was increased to $80 \mathrm{mg}$ once daily. Computed tomography did not show signs of stroke. Creatine kinase (CK) and myoglobin levels $(1,276$ $\mu \mathrm{g} / \mathrm{L}$ ) were increased, and ICI-induced myositis was therefore suspected. In addition, a gradual decrease in creatinine levels was observed (Fig. 1c). Antibodies against acetylcholine receptor $(2.6 \mathrm{nmol} / \mathrm{L})$ and titin were present, indicating MG. In addition, albumin (516 mg/L) was present in the cerebrospinal fluid. On day 34, the patient was unable to sit up, had pain in his neck and shoulders, had developed severe dysarthria and dysphagia, and could not attain saturation without oxygen. The patient had absent reflexes in the biceps, brachioradialis, triceps, and patellar and Achilles tendons. The same day, he was transferred to the intensive care unit; he was intubated the following day due to suspected immunological involvement of the intercostal musculature. The patient was given methylprednisolone $1 \mathrm{~g} /$ $\mathrm{kg}$ ) during 3 days and intravenous immunoglobulins. On day 37, he was given infliximab (5 $\mathrm{mg} / \mathrm{kg}$ ). On day 38 , the patient felt better and had better muscle strength in his hands. On day 39 , the patient developed carbon dioxide retention and needed noninvasive ventilation, and he developed sinus bradycardia. He eventually died on day 39. 


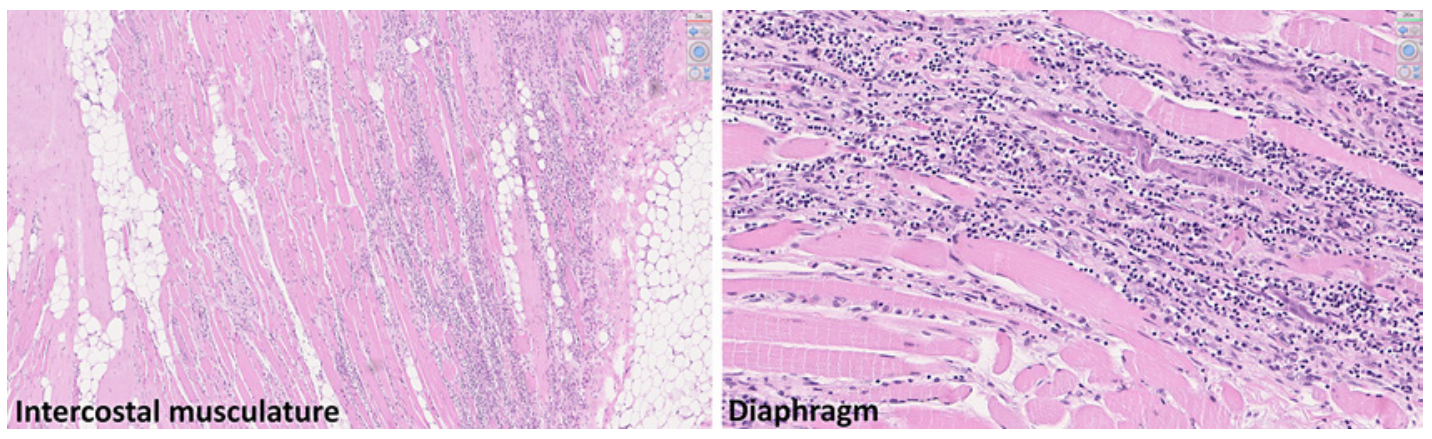

Fig. 2. Biopsies taken at autopsy from the intercostal musculature $(\times 200)$ and diaphragm $(\times 50)$ showing a pronounced inflammatory infiltrate of the skeletal muscle.

Autopsy showed a significant stenosis of the right coronary artery but no fibrosis or signs of recent myocardial infarction. The tongue was softened. No surgical complication after hemicolectomy was observed. A $50 \times 60 \mathrm{~mm}$ metastasis and 3-4 up to 5-mm metastases were observed in the right liver lobe. Microscopical examination of the intercostal musculature, diaphragm, cervical musculature and tongue showed pronounced inflammatory infiltration of lymphocytes, occasional plasma cells and granulocytes, and fibrosis, consistent with a pronounced myositis (Fig. 2). Biopsies from the heart showed fibrosis in one area, consistent with myocardial infarction. In a small area of the heart, an inflammatory infiltrate was observed, with similarities to the inflammatory infiltrates in the skeletal musculature. In the liver, microscopical examination did not show metastases from colorectal cancer but instead a hepatocellular cancer (HCC) positive for hepatocytes and negative for glypican, CDX2, CK20 and CK7. In addition, fibrosis stage 2-3 according to Batts and Ludwig in the porta field was observed. The cause of death was determined as respiratory insufficiency due to polymyositis.

\section{Discussion}

Despite the fact that our patient was given high doses of corticosteroids, intravenous immunoglobulins, infliximab and intensive care (according to clinical practice), the patient succumbed to irAEs. Autopsy displayed ICI-induced autoimmune involvement of both skeletal muscles and cardiac muscles. Haddox et al. [4] reported a case of autopsy-verified pembrolizumab-induced bulbar myopathy, myocarditis, T-cell infiltration of the diaphragm and respiratory insufficiency. Not only did our patient show signs of myositis including muscular pain and weakness, but laboratory testing also indicated myositis with increased levels of CK and myoglobin. We initially suspected hepatitis due to an elevation of AST and ALT. However, there are many extrahepatic sources of AST and ALT - for example, skeletal and cardiac muscles [5].

Shi et al. [6] reported that neurological irAEs occurred in $2.6 \%$ of patients under PD-1 targeted therapy. In that study, almost half of the cases had encephalitis and less than $0.5 \%$ neuropathy. Suzuki et al. [7] showed that $0.12 \%$ of nivolumab-treated patients developed MG. In our case, antibodies against acetylcholine receptor and titin were observed, showing that the patient also had developed MG [8]. Antibodies against acetylcholine receptor are common in ICI-induced MG [9]. In patients developing neurological irAEs, it is common with overlap syndromes, that is, with combined syndromes of myositis, MG and neuropathy [10]. It is also 
common with concomitant myositis and myocarditis and high levels of serum CK along with ICI-induced MG $[3,7]$. Positivity for anti-titin has previously been reported for pembrolizumab-induced MG combined with necrotizing myositis [11]. Clinical signs of MG were difficult to identify in the predominant clinical picture of polymyositis in our patient. In addition, albumin was present in the cerebrospinal fluid, which could be consistent with the Guillain-Barré syndrome [12]. As mentioned, in the predominant clinical picture of polymyositis, absence of reflexes was difficult to separate from a general detriment to muscle force. We were not able to perform neurography as well.

To our surprise, autopsy revealed no presence of colorectal metastases but instead the presence of HCC. The HCC seemed to have responded partly to pembrolizumab therapy. Pembrolizumab has shown efficacy against HCC, and it was approved in 2017 for solid tumors with MMR-D/MSI-H including HCC and in 2018 for advanced HCC [13]. ICIs have been suggested to be used against PD-1/PD-L1-expressing HCC [14]. Our patient had two primary cancers, of which the colorectal tumor was MMR-D. Lynch syndrome is a condition due to germline mutations in MMR genes. We do not know whether our patient had Lynch syndrome; however, patients with Lynch syndrome are not only at risk of developing colorectal cancer and endometrial cancer, but also at an increased risk of developing other primary cancers including tumors in the hepatobiliary tract [15].

In conclusion, ICI-induced myositis may be accompanied by neurological irAEs which may have a subtle course initially but may take a dramatic and fatal course eventually. In patients experiencing muscle pain and weakness after receiving immunotherapy, myositis should be suspected.

\section{Statement of Ethics}

This case report was accomplished in accordance with the Declaration of Helsinki. The patient's wife has given informed consent to publish this case report.

\section{Conflict of Interest Statement}

The authors declare no conflict of interest.

\section{Funding Sources}

No funding was received for this case report.

\section{Author Contributions}

D.G. and L.N. were responsible for the design of the case report, for acquisition and interpretation of the data, and for drafting of the manuscript. D.G. was responsible for the oncological care of the patient, H.B. for the neurological care of the patient, and A.F. for immunohistochemistry, autopsy and the pathology report. All authors contributed to and approved the final draft of the manuscript.

\section{Karger 'ह}




\section{Case Reports in Oncology}

\begin{tabular}{l|l}
\hline Case Rep Oncol 2020;13:1252-1257 \\
\hline DOI: 10.1159/000510740 & $\begin{array}{l}\text { @ 2020 The Author(s). Published by S. Karger AG, Basel } \\
\text { www.karger.com/cro }\end{array}$ \\
\hline
\end{tabular}

Giglio et al.: Fatal Polymyositis Induced by Pembrolizumab

\section{References}

1 Boussiotis VA, Chatterjee P, Li L. Biochemical signaling of PD-1 on T cells and its functional implications. Cancer J. 2014 Jul-Aug;20(4):265-71.

2 Hottinger AF. Neurologic complications of immune checkpoint inhibitors. Curr Opin Neurol. 2016 Dec;29(6): 806-12.

3 Vermeulen L, Depuydt CE, Weckx P, Bechter 0, Van Damme P, Thal DR, et al. Myositis as a neuromuscular complication of immune checkpoint inhibitors. Acta Neurol Belg. 2020 Apr;120(2):355-64.

4 Haddox CL, Shenoy N, Shah KK, Kao JC, Jain S, Halfdanarson TR, et al. Pembrolizumab induced bulbar myopathy and respiratory failure with necrotizing myositis of the diaphragm. Ann Oncol. 2017 Mar 1;28(3):673-5.

5 Wroblewski F. The clinical significance of alterations in transaminase activities of serum and other body fluids. Adv Clin Chem. 1958;1(2):313-51.

6 Shi S, Jaoube JA, Kanwar R, Jin MC, Amorin A, Varanasi V, et al. Neurological adverse effects due to programmed death 1 (PD-1) inhibitors. J Neurooncol. 2020 Jun;148(2):291-7.

7 Suzuki S, Ishikawa N, Konoeda F, Seki N, Fukushima S, Takahashi K, et al. Nivolumab-related myasthenia gravis with myositis and myocarditis in Japan. Neurology. 2017 Sep 12;89(11):1127-34.

8 Lazaridis K, Tzartos SJ. Autoantibody specificities in myasthenia gravis; implications for improved diagnostics and therapeutics. Front Immunol. 2020;11:212.

9 Becquart O, Lacotte J, Malissart P, Nadal J, Lesage C, Guillot B, et al. Myasthenia gravis induced by immune checkpoint inhibitors. J Immunother. 2019 Oct;42(8):309-12.

10 Möhn N, Beutel G, Gutzmer R, Ivanyi P, Satzger I, Skripuletz T. Neurological immune related adverse events associated with nivolumab, ipilimumab, and pembrolizumab therapy - review of the literature and future outlook. J Clin Med. 2019 Oct;8(11):1777.

11 Onda A, Miyagawa S, Takahashi N, Gochi M, Takagi M, Nishino I, et al. Pembrolizumab-induced ocular myasthenia gravis with anti-titin antibody and necrotizing myopathy. Intern Med. 2019 Jun;58(11):1635-8.

12 Ropper AH. The Guillain-Barré syndrome. N Engl J Med. 1992 Apr;326(17):1130-6.

13 Zhu AX, Finn RS, Edeline J, Cattan S, Ogasawara S, Palmer D, et al. Pembrolizumab in patients with advanced hepatocellular carcinoma previously treated with sorafenib (KEYNOTE-224): a non-randomised, open-label phase 2 trial. Lancet Oncol. 2018 Jul;19(7):940-52.

14 Moris D, Rahnemai-Azar AA, Zhang X, Ntanasis-Stathopoulos I, Tsilimigras DI, Chakedis J, et al. Program death-1 immune checkpoint and tumor microenvironment in malignant liver tumors. Surg Oncol. 2017 Dec; 26(4):423-30.

15 Win AK, Lindor NM, Young JP, Macrae FA, Young GP, Williamson E, et al. Risks of primary extracolonic cancers following colorectal cancer in lynch syndrome. J Natl Cancer Inst. 2012 Sep 19;104(18):1363-72. 